\section{Paradoxical retinal motions during head movements: Apparent motion without equivalent apparent displacement}

\author{
WAYNE L. SHEBILSKE \\ and DENNIS R. PROFFITT \\ University of Virginia, Charlottsville, Virginia
}

Passengers in moving vehicles often see an interesting phenomenon when they fixate one of several objects that are at different distances. Looking sideways out the window, they may notice that all objects closer than the fixated object appear to move in a direction opposite to their movement, whereas all objects farther than the fixated object appear to move in the direction in which they are traveling. You can see similar apparent motions by holding your index fingers at different distances while swinging your head back and forth. When you look at the far finger, the near one appears to move against the direction of head movements; when you look at the near finger, the far one appears to move with the direction of head movement. The term "motion parallax" is often used in referring to these and other apparent motions that are concomitant with lateral head movements (e.g., Coren, Porac, \& Ward, 1979; Gibson, Gibson, Smith, \& Flock, 1959; Shebilske \& Proffitt, 1981). Gogel, however, prefers the term "apparent concomitant motion." This paper will adopt Gogel's term to avoid clouding issues in an ongoing debate that contends issues much deeper than terminology.

On one side of the debate, Gogel $(1980,1981,1982)$ argues for an apparent-distance/pivot-distance hypothesis, according to which the apparent motions occur because people underestimate the distance between objects. On the other side, Shebilske and Proffitt (1981) argue for a paradoxical-motion hypothesis, according to which apparent motions occur because people experience a paradoxical sensation of retinal motions. This paper will analyze these two hypotheses and their implications for visual-motor coordination, motion perception, and distance perception.

One issue in the debate involves a distinction between apparent motion per se and apparent displacement or change in target location. The distinction is important because people sometimes experience paradoxical motion, which is a perception resulting

The preparation of this paper was supported by NIE Grant R01EY02291-03, awarded to Wayne L. Shebilske, and NICHD Grant HD-16195, awarded to Dennis R. Proffitt and Bennett I. Bertenthal. Requests for reprints should be sent to Wayne L. Shebilske, Department of Psychology, Gilmer Hall, University of Virginia, Charlottesville, Virginia 22901. in an incompatible relationship between a target's apparent motion and its apparent displacement (or lack of displacement). V. Kries (1962) credited S. Exner with distinguishing apparent motion and displacement and with establishing the existence of paradoxicalmotion perception. The apparent concomitant motions observed in the moving vehicle and finger examples may be instances of perceiving paradoxical motions. Shebilske and Proffitt (1981) were therefore critical of studies that collected only measures of apparent displacement and then generalized about apparent concomitant motion (e.g., Gogel, 1980). The present paper will carry that criticism a step farther by using Gogel's (1982) data to illustrate the paradoxical relationship between measures of perceived apparent motion and displacement.

Another issue in the debate concerns the effect of retinal image motions that occur during lateral head movements whenever targets are at distances other than the distance to an observer's fixation point. Thus, retinal image movements will occur even with a single target if an observer converges his or her eyes in front or in back of the target. Several researchers have suggested that such convergence errors may be responsible for apparent concomitant motions (Owens \& Leibowitz, 1976, 1980; Post \& Leibowitz, 1982; Shebilske, 1977). Gogel (1982) tested this possibility by measuring convergence during lateral head movements. He found that convergence errors were not necessary for apparent concomitant motion of a single target. Subjects who overestimated a target's distance saw apparent concomitant motion of a stationary target even though they had little or no convergence error. This illusory movement was nonparadoxical in the sense that there was good agreement between measures of apparent motion and displacement perceptions. Gogel's results mean that retinal image motion is not necessary for apparent concomitant motion, but they do not rule out the possibility that retinal image motions contribute to the phenomenon. In fact, this paper will use Gogel's (1982) data to argue that when retinal image motions are present they contribute to apparent concomitant motion.

\section{Contrasting Predictions}

Important differences between the apparentdistance/pivot-distance hypothesis and the paradoxical-motion hypothesis are brought into focus when both hypotheses are used to generate contrasting predictions about perceived apparent concomitant motion in two of Gogel's critical conditions. These two situations are illustrated in Figure 1.

Drawing A illustrates a condition in which subjects fixate a point (FP) that is beyond a target (T); they 


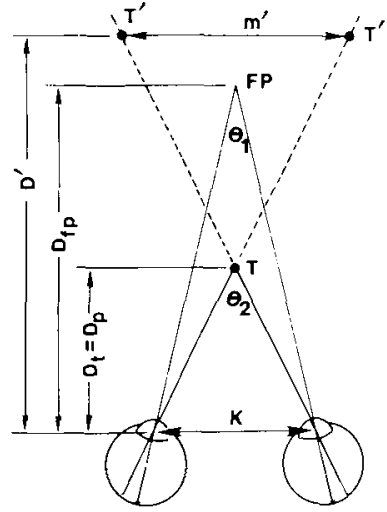

A

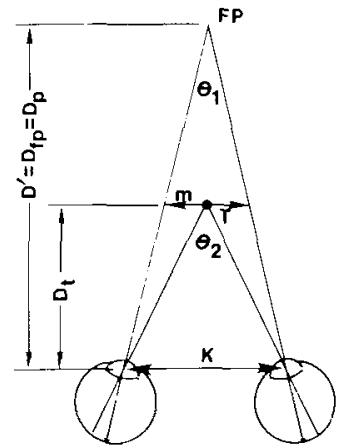

B
Figure 1. Retinal motion produced by lateral head movement in two viewing conditions. For simplicity, only one translating eye is represented in each condition. The analysis also applies to binocular conditions, however, because comparable motions occur in each eye. In conditions $A$ and $B$, the tanget is at $T$, the fixation point is at FP, and the eye moves laterally along line $K$. The distance to the target is $D_{t}$. The point distance, $D_{p}$, is the distance from the observer to a hypothetical point around which the direction to the target pivots as the head moves laterally. The distance to fixation point is $D_{f p}$. The apparent distance to the target is $D^{\prime}$. The angle through which the eye rotates is $\theta_{1}$. The angle through which the retinal image of $T$ will traverse with respect to an eye that does not rotate is $\theta_{2}$. The angular extent of retinal image motion is $\theta_{2}$ minus $\theta_{1}$. In condition $A$, the target appears to be at the $T^{\prime}$ positions before and after each lateral head movement. The target appears to move along path $\mathrm{m}^{\prime}$. In condition $B$, subjects move the target along line $m$ to null the apparent concomitant motion that they otherwise would have seen.

see the target farther away than it actually is [i.e., apparent distance $\left(D^{\prime}\right)$ is greater than the target distance $\left.\left(D_{t}\right)\right]$, and they see apparent concomitant motion of the target $\left(\mathrm{m}^{\prime}\right)$ when they move their heads laterally along line $K$. The pivot distance $\left(D_{p}\right)$ is the distance from the observer to a hypothetical point around which the direction to the target pivots as the head moves laterally. Gogel took four kinds of measures in this condition:

(1) He measured convergence distance, which allows one to compute retinal motion.

(2) He measured the distance of subjects' pointing responses when they were asked to point under the target's apparent location at the extreme right and left head positions (pointing procedure).

(3) He also used the pointing procedure to measure the apparent lateral change in the target's location before and after head movements.

(4) And he measured the apparent lateral change in the target's location before and after head movements by having subjects set the visual separation of comparison posts to match the apparent lateral change in target location (hand adjustment procedure).

Notice that all measures of $\mathrm{m}^{\prime}$ were measures of displacement perception. Gogel did not measure per- ceived apparent motion in situation $\mathbf{A}$. He did, however, measure apparent-motion perception in situation $B$, in which subjects fixated a point (FP) that was beyond a target $(T)$; they saw the target's apparent distance $\left(D^{\prime}\right)$ as being equal to the distance to their fixation point $\left(D_{\mathrm{fp}}\right)$, and they moved the target along line $\mathrm{m}$ to null the apparent concomitant motion that would otherwise have been seen when they moved their heads laterally along line $\mathrm{K}$.

For situation A, the apparent-distance/pivotdistance hypothesis states that $\mathrm{m}^{\prime}$ is determined by the ratio $D^{\prime} / D_{p}$ for a constant value of $K$. Accordingly, Gogel (1982) gave Equation 1 as a prediction (illustrated in his Figure 1B):

$$
m^{\prime}=K\left(1-D^{\prime} / D_{p}\right) \text {. }
$$

Gogel listed two additional equations that are useful in applying the apparent-distance/pivot-distance hypothesis to situation $\mathrm{A}$. These equations can be simplified with respect to drawing $A$ as follows:

$$
\begin{gathered}
m^{\prime}=\left(K D_{t}-K D^{\prime}\right) / D_{t} \\
D^{\prime}=D_{t}\left(K-m^{\prime}\right) / K
\end{gathered}
$$

For situation B, Equation 4 is a prediction of null adjustments $(\mathrm{N})$ based on the apparent-distance/ pivot-distance hypothesis (illustrated in Gogel's Figure 1C):

$$
N=m=K-\frac{\left(K D_{1}\right)}{D^{\prime}}
$$

Equation 5 follows from Equation 4:

$$
\mathrm{D}^{\prime}=\mathrm{KD}_{\mathrm{t}} /(\mathrm{K}-\mathrm{m}) \text {. }
$$

The relationship between these various predictions is important. Especially critical is the relationship between Equations 3 and 4, which suggests that one should obtain equivalent measures of $D^{\prime}$ through perceived displacement measures of $\mathrm{m}^{\prime}$ or through perceived apparent-motion measures of $m$ without taking retinal motions into account.

This predicted relationship between apparentmotion and displacement-perception measures and their corresponding equations is at odds with the paradoxical-motion hypothesis, which states that retinal motions that are concomitant with lateral head movements cause apparent target motions without causing a change in the target's apparent location before and after the head movement. As we stated in an earlier paper (Shebilske \& Proffitt, 1981), the apparent motion perception related to retinal motions should combine vectorially with other sources of motion perception. However, in contrast to sources of nonparadoxical motion, which influence both mo- 
tion and displacement perception, paradoxical retinal motions should affect apparent-motion perception without influencing displacement perception. To test the paradoxical-motion hypothesis, one must compare measures of retinal image motion, displacement perception at the beginning and end of head movements, and apparent motion perception during head movements.

To compute the angular extent of retinal image motion (RIM) in situations A or B of Figure 1, one must first calculate the angle through which the eyes rotate $\left(\theta_{1}\right)$ and the angle through which the retinal image of $T$ will traverse with respect to an eye that does not rotate $\left(\theta_{2}\right)$. These values can be computed with Equations 6 and 7:

$$
\begin{aligned}
& \tan \frac{\theta_{1}}{2}=K / 2 D_{\mathrm{fp}}, \\
& \tan \frac{\theta_{2}}{2}=K / 2 D_{1} .
\end{aligned}
$$

One can then compute RIM with Equation 8:

$$
\operatorname{RIM}=\left(\theta_{2}-\theta_{1}\right) .
$$

In applying the paradoxical-motion hypothesis to situation B of Figure 1, it is also important to compute the lateral motion of $T$ required to null retinal image motion (NRIM), which is given by Equation 9:

$$
\mathrm{NRIM}=2 \mathrm{D}_{\mathrm{t}} \tan \frac{\mathrm{RIM}}{2} .
$$

Equation 9 allows one to make precise comparisons of predictions based on the apparent-distance/ pivot-distance hypothesis and those based on the paradoxical-motion hypothesis. Retinal motions should not influence perceived displacement according to either hypothesis, so Equations 1-3 should make accurate predictions in situations that include or do not include retinal motions. Retinal motions should add to apparent-motion perception, however, according to the paradoxical-motion hypothesis. As a result, Equation 4 should give accurate predictions only when no retinal motion is present. Otherwise, according to the paradoxical motion hypothesis, better predictions will be given by Equation 10:

$$
\mathbf{N}=\mathbf{m}+\mathbf{N R I M} \text {. }
$$

Similarly, Equation 5 should give accurate predictions only when no retinal motion is present. Otherwise, better predictions will be given by Equation 11 according to the paradoxical motion hypothesis.

$$
D^{\prime}=K D_{t} /(K-m-N R I M) \text {. }
$$

In short, the contrasting predictions bring two issues into focus: (1) the relationship between apparent motion and displacement perceptions and (2) the effects of retinal motions. The apparentdistance/pivot-distance hypothesis suggests that perceived apparent motion and displacement will be comparable and that retinal motions will have no effect. In contrast, the paradoxical-motion hypothesis suggests that perceived apparent motion and displacement will not agree when retinal motions are present because retinal motions influence apparentmotion perception without affecting perceived displacement.

\section{Tests of the Contrasting Predictions}

The predictions based on the paradoxical motion hypothesis provide a better account of the data in Table 1, which comes from Gogel's (1982) Experiment 1 . Estimates of apparent distance $\left(D^{\prime}\right)$ to a stationary target are given as a function of convergence distance. These estimates were obtained from: (1) the distance of pointing responses, (2) the average of two procedures for measuring perceived displacement (Equation 3 applied to results of the pointing procedure and the hand-adjustment procedure), and

\begin{tabular}{|c|c|c|c|}
\hline Observer & $\begin{array}{c}\text { Distance of } \\
\text { Pointing Responses }\end{array}$ & $D^{\prime *}$ & $D^{\prime * *}$ \\
\hline \multicolumn{4}{|c|}{$C D=25$} \\
\hline $\begin{array}{l}1 \\
2 \\
3 \\
4 \\
5 \\
6\end{array}$ & $\begin{array}{l}39.3 \\
32.2 \\
28.6 \\
37.0 \\
34.5 \\
27.3\end{array}$ & $\begin{array}{l}37.9 \\
35.2 \\
36.8 \\
32.4 \\
43.2 \\
35.0\end{array}$ & $\begin{array}{l}43.1 \\
44.8 \\
55.8 \\
48.8 \\
62.2 \\
33.5\end{array}$ \\
\hline Mean & 33.1 & 36.8 & 48.0 \\
\hline \multicolumn{4}{|c|}{$\mathrm{CD}=40$} \\
\hline $\begin{array}{l}1 \\
2 \\
3 \\
4 \\
5 \\
6\end{array}$ & $\begin{array}{l}46.4 \\
42.4 \\
38.7 \\
51.2 \\
39.6 \\
43.2\end{array}$ & $\begin{array}{l}48.2 \\
51.0 \\
41.3 \\
43.2 \\
43.8 \\
45.2\end{array}$ & $\begin{array}{l}96.0 \\
64.6 \\
78.6 \\
88.3 \\
95.8 \\
47.0\end{array}$ \\
\hline Mean & 43.6 & 45.4 & 78.4 \\
\hline
\end{tabular}
(3) Equation 5 applied to the results of the null adjustment measures of perceived apparent concomitant motions. We tested differences between means

Table 1 Results from Gogel (1982) Experiment 1

Note-Apparent Distance $\left(D^{\prime}\right)$ to a stationary target in centimeters as a function of convergence distance (CD). Estimates of $D^{\prime}$ were obtained from the distance of pointing responses, from the average of two procedures for measuring apparent change in target location before and after head movements, and from null adjustment measures of apparent motion during head movement. ${ }^{*} D^{\prime}$ computed from apparent changes in target location. ${ }^{* *} D^{\prime}$ computed from measures of apparent motion. 
in Table 1 with matched-pair $t$ tests, employing a criterion of $p<.01$ on a one-tail test. We found a pattern of results that is in opposition to the apparentdistance/pivot-distance hypothesis and in support of the paradoxical motion hypothesis.

Both hypotheses make the same predictions at a convergence distance of $25 \mathrm{~cm}$, since Gogel's Experiment 2 showed that convergence was on target for all practical purposes in that condition. Specifically, the two hypotheses predict that all three kinds of measures should yield equivalent estimates of $D^{\prime}$. The results support the mutual prediction, since none of the differences between means were significant.

The two hypotheses make different predictions at a convergence distance of $40 \mathrm{~cm}$. Considerable retinal motion occurred in this condition, since the target was at $25 \mathrm{~cm}$ while convergence was at about $40 \mathrm{~cm}$. (Gogel's Experiment 2 showed that convergence deviated by a negligible amount from $40 \mathrm{~cm}$.) The retinal motion should not change the pattern of results, according to the apparent-distance/pivot-distance hypothesis. In other words, this hypothesis predicts that all three kinds of measures should yield equivalent estimates of $\mathrm{D}^{\prime}$. In contrast, the paradoxicalmotion hypothesis predicts that the retinal motion should inflate the measures of perceived apparent motion without affecting the measures of displacement perception. In other words, our hypothesis predicts that the estimates of $D^{\prime}$ that are based on apparent motion measures should be inflated, while the estimates of $D^{\prime}$ based on the other two measures should remain equivalent to one another. The results clearly oppose the apparent-distance/pivot-distance hypothesis and strongly support the paradoxicalmotion hypothesis. Specifically, estimates based on apparent-motion-perception measures $\left(D^{\prime}=78.4\right)$ were significantly higher than those based on perceived-displacement measures $\left(D^{\prime}=45.4\right)[t(5)=$ $3.95, \mathrm{p}<.01]$, whereas distance of pointing $\left(\mathrm{D}^{\prime}=\right.$ 43.6) did not differ significantly from the estimates based on perceived-displacement measures $[t(5)=$ $0.81, \mathrm{p}>1$ ].

Figure 2 illustrates the pattern of results in another way. It shows the predictions that Equation 4 makes for $\mathbf{N}=\mathbf{m}$ when $D^{\prime}$ estimates are taken from distance of pointing responses and when $\mathrm{D}^{\prime}$ estimates are țaken from measures of displacement perception. It also shows the obtained $\mathrm{N}$ and the obtained $\mathrm{m}$ according to Equation 10 . The obtained $N(14 \mathrm{~cm})$ was significantly larger than the predictions based on distance of pointing $(8.9 \mathrm{~cm})[\mathrm{t}(5)=5.15, \mathrm{p}<.01]$ and significantly larger than the predictions based on the perceived displacement measures $(9.4)[t(5)=4.41$, $p<.01]$. The results suggest, therefore, that Equation 4 is wrong in assuming that $N=m$. The results also suggest that Equation 10 is correct in suggesting that $\mathbf{N}=\mathbf{m}+\mathbf{N R I R}$. When $\mathrm{m}$ is computed according to Equation $10(6.8 \mathrm{~cm})$, it is not significantly differ-

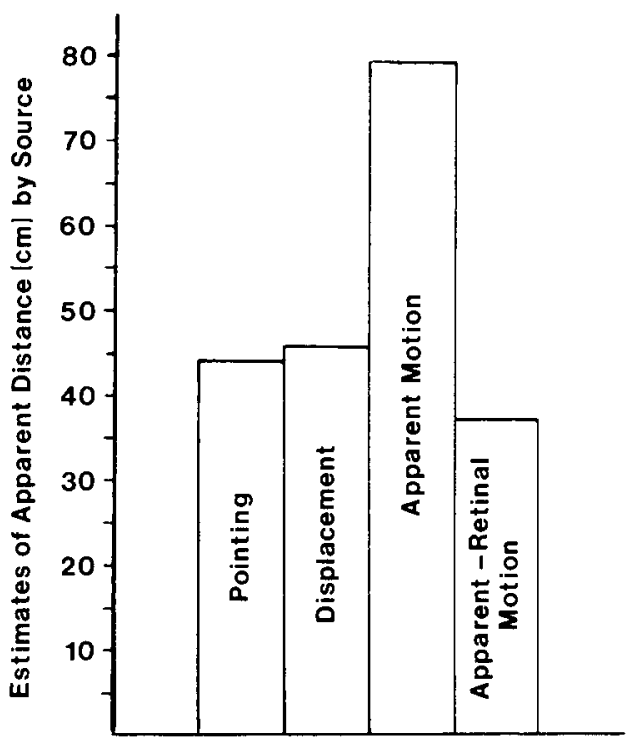

Figure 2. Various estimates of apparent motion show that the obtained amount of null adjustment (third bar) is more than predicted by Equation 4 and $D^{\prime}$ estimates from the distance of pointing responses (first bar) or by Equation 4 and $D^{\prime}$ estimates from displacement measures (second bar). The null adjustment measures agree with the other measures better when the retinal motion component is subtracted, as specified by Equation 11 (fourth bar).

ent from the pointing prediction of $\mathrm{m}[\mathrm{t}(5)=2.16$, $p>.01]$ or from the perceived displacement prediction of $\mathrm{m}[\mathrm{t}(5)=2.67, \mathrm{p}>.01]$.

Figure 3 shows the results another way. The first three bars illustrate results that we have already analyzed in Table 1. They are: (1) the distance of pointing responses, (2) $D^{\prime}$ computed from Equation 3 and measures of perceived displacement, and (3) D' computed from Equation 5 and measures of apparent motion perception, which gave significantly higher estimates than the other two procedures, as mentioned earlier. The fourth bar shows that Equation 11 gave much better estimates of $D^{\prime}$. In fact, the estimates from Equation 11 were not significantly different from the estimates from Equation 3 and measures of perceived displacement $[t(5)=3.05, p>.01]$. Thus, Equation 11, which is based on the paradoxical motion hypothesis gives better predictions than Equation 5, which is based on the apparent-distance/ pivot-distance hypothesis.

\section{Implications}

Our analysis of Gogel's (1982) results has implications for visual-motor coordination, motion perception, and distance perception. We will take up each of these in turn.

Visual-motor coordination. Two aspects of visualmotor coordination are: visual direction constancy, which is the relative invariance of an object's apparent egocentric direction despite changes in the ob- 


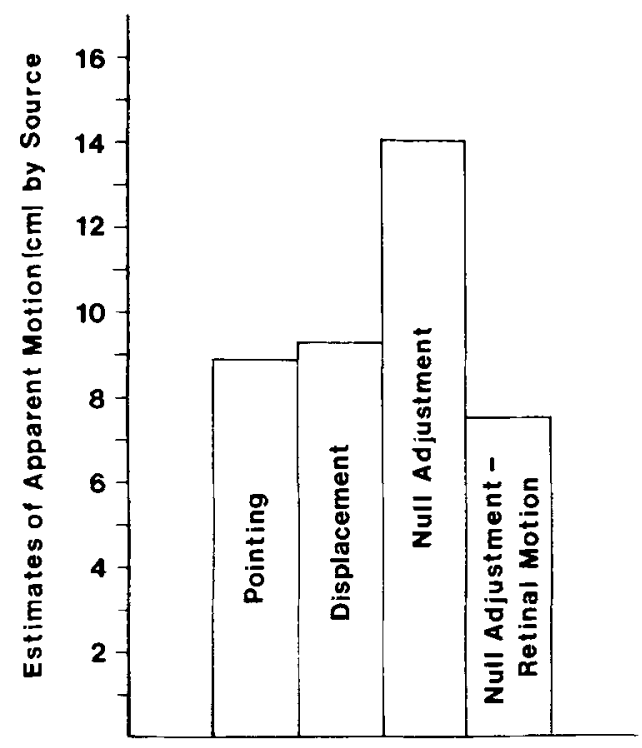

Figure 3. Various estimates of apparent distance $\left(D^{\prime}\right)$ show that the estimates based upon Equation 5 and apparent motion (third bar) are higher than the distance of the pointing response (first bar) and higher than estimates based upon Equation 3 and displacement measures (second bar). The apparent motion measures agree with the other measures better when the retinal motion component is subtracted, as specified by Equation 11 (fourth bar).

ject's oculocentric direction, and visual position constancy, which is the relative invariance of an object's apparent spatial location despite changes in the object's egocentric direction. Neither constancy is perfect, and the failures of each reveal properties of underlying processes. The apparent streaking of a luminous stimulus viewed against a dark background (Hill, 1972; Matin, Clyner, \& Matin, 1972) is an example of a failure of visual direction constancy. Apparent concomitant motion during lateral head movements is an example of a failure of visual position constancy. Visual direction constancy has a dynamic apparent-motion-perception component and a static perceived-location component. These two components fail independently, thereby suggesting that they are determined by separate processes (Shebilske, 1977). Similarly, the present analysis suggests that perceiving apparent concomitant motion fails independently of displacement perception. Thus, these two components may also be based upon separate processes.

Apparent concomitant motions seen in everyday situations, such as riding in a car, are clearly failures of the apparent-motion component of visual position constancy. Determining whether they also include failures of the apparent-displacement component will require field experiments. Gogel's research shows that errors in perceived distance can affect perceived apparent concomitant motion. Based on this research, Gogel concluded that "the many instances of appar- ent motion obtained when stationary objects are viewed with a moving head in visually rich environments reflect the presence of errors in perceived distance, [and that] ... although the three-dimensional world seems (subjectively) to be well structured in distance, the perception of depth or distance is often in error" (Gogel, 1980, p. 155). These conclusions are premature according to the present analysis, which suggests that factors other than distance illusions influence apparent-concomitant-motion perception. People may experience apparent concomitant motions in everyday situations, even though they see distances accurately.

According to Gogel, suggesting that stationary objects "both appear to move and are perceived correctly in distance is like having one's cake and eating it too" (Gogel, 1981, p. 176). The contribution of retinal image motions to apparent-motion perception without a contribution to displacement perception is also a paradox like having one's cake and eating it too. So we agree with Gogel's comment, but we disagree with its intended meaning that apparent concomitant motion without distance illusions is impossible. We believe it is possible, and the superiority of Equation 10 over Equation 4 and of Equation 11 over Equation 5 in the present analysis supports our position.

Motion perception. We question the priority of perceived distance, not only for perceiving apparent concomitant motion, but also for perceiving motion in general. Gogel (1980) suggested that without some perception of distance, the perception of motion would be impossible. We stand by an earlier statement that, in certain situations (e.g., Gogel, 1980), perceived motions can be based solely on motion information and principles of perceptual organization that make no use of distance information. Examples of such principles are Cutting and Proffitt's (1982) and Restle's (1979) minimum principles. In Gogel's (1980) experiment, a Polaroid stereoscope manipulated binocular disparity information in order to alter perceived slant of a line. The stereoscope thereby varied the binocular disparity of retinal motions, which, together with other proximal information, determined an indefinite set of possible events, all but one of which included two motion parameters: translation and rotation. Both Cutting and Proffitt's and Restle's minimum principles would select the one nontranslating alternative, which would be the rotations that Gogel's subjects saw. Gogel's (1980) experiment provides no way of ruling out the possibility that perceptions were based on a minimum principle operating solely on motion information, including the binocular disparity of the moving images. In contrast, Gogel's (1982) experiment rules out the proposed minimum principles as the bases for the motions seen in that experiment, since both approaches would have predicted no motion. Future research 
is faced with the challenge of determining whether some other minimum principle could explain the perceived motions or whether a single point viewed under darkroom conditions is outside the boundary conditions for the operation of all minimum principles. A more general challenge will be formulating a theory that will account for all cases of motion perception. If such a theory is ever put forth, it will probably not include the general assertion of the priority of perceived distance for perceiving motion, since the present analysis suggests that perceived distance is not always involved in the apparent concomitant motion of physically stationary objects.

Distance perception. Gogel uses Equations 3 and 5 to compute indirect measures of apparent distance from data on apparent concomitant motions. He uses these indirect measures to test important hypotheses about various aspects of space perception, such as the relationship between apparent size and apparent distance. The present analysis suggests that he would do better to use Equations 3 and 11. Despite the superiority of Equation 11 over Equation 5, however, we do not recommend it as a general measure of apparent distance. Although a good geometer can make a strong case for the geometric logic of using one value of apparent distance for seeing various aspects of space (e.g., Gogel, 1973), we are not convinced that perceptual processes strictly follow a geometric logic. Perceptual processes may, for instance, use different values of apparent distance for different purposes, even though geometry dictates that there is only one value. Suppose, for example, that an observer viewed the same tree with four different purposes in mind: (1) to throw a stone next to it, (2) to cut a rope equal to the length of a branch, (3) to cut a rope to span the depth between a near and far branch, and (4) to compute the motion path of a swaying branch. The four purposes call for the perception of absolute distance, size, depth, and motion, respectively. The geometry of the situation could indicate that the same distance value is appropriate for all four perceptions, but we would be concerned that the observer might attend to different distance information, depending upon the purpose for computing distance, and thereby register different values of distance for each purpose. This possibility does not imply that an observer simultaneously perceives the tree at different distances, but it does suggest that an observer might register different distances for different purposes. In light of our concern about the perceptual system deviating from geometric logic, we are skeptical of attempts to treat geometrically based equations as axioms. We view "perceptual equations" (Gogel, 1973), "algorithms" (Ebenholtz, 1977), and "taking-into-account" for- mulas (Epstein, 1973; Rock, 1977) not as axioms, but as theories to be tested. Experiments with precisely done converging operations, such as those reported by Gogel (1982), will be valuable in testing those theories.

\section{REFERENCES}

Coren, S., Porac, C., \& Ward, L. M. Sensation and perception. New York: Academic Press, 1979.

Cuting, J. E., \& Proffit, D. R. The minimum principle and the perception of absolute, common, and relative motions. Cognitive Psychology, 1982, 14, 211-246.

Erenholtz, S. M. The constancies in object orientation: An algorithm processing approach. In W. Epstein (Ed.), Stability and constancy in visual perception: Mechanisms and processes. New York: Wiley, 1977.

EPSTE in, W. The process of "taking-into-account" in visual perception. Perception, 1973, 2, 267-285.

Gibson, E. J., Gibson, J. J., Smith, O. W., \& Flock, H. Motion parallax as a determinant of perceived depth. Journal of Experimental Psychology, 1959, 58, 40-51.

Gogel, W. C. The organization of perceived space I: Perceptual interactions. Psychologische Forschung, 1973, 36, 195-221.

Gogel, W. C. The sensing of retinal motion. Perception \& Psychophysics, 1980, 28, 155-163.

Gogel, W. C. Perceived depth is a necessary factor in apparent motion concomitant with head motion: A reply to Shebilske and Proffitt. Perception \& Psychophysics, 1981, 29, 173-177.

GoGel, W. C. Analysis of the perception of motion concomitant with a lateral motion of the head. Perception \& Psychophysics, 1982, 32, 241-250.

Hill, A. L. Direction constancy. Perception \& Psychophysics, 1972, 11, 175-178.

Matin, E., Clyner, A. B., \& Matin, L. Metacontrast and saccadic suppression. Science, 1972, 178, 179-182.

OWens, D. A., \& Leibowitz, H. W. Oculomotor adjustments in darkness and the specific distance tendency. Perception \& Psychophysics, 1976, 20, 2-9.

OWEns, D. A., \& Leibowitz, H. W. Accommodation, convergence and distance perception in low illumination. American Journal of Optometry and Physiological Optics, 1980, 57, 540-550.

Post, R. B., \& Leibowitz, H. W. The effects of convergence on the vestibula-ocular reflex and implications for perceived movement. Vision Research, 1982, 22, 461-465.

Restle, F. Coding theory of the perception of motion configurations. Psychological Review, 1979, 86, 1-24.

Rock, I. In defense of unconscious inference. In W. Epstein (Ed.), Stability and constancy in visual perception: Mechanisms and processes. New York: Wiley, 1977.

Sherilske, W. L. Visuomotor coordination in visual direction and position constancies. In W. Epstein (Ed.), Stability and constancy in visual perception: Mechanisms and processes. New York: Wiley, 1977.

Shebilske, W. L., \& Proffitt, D. R. The priority of perceived distance for perceiving motion has not been demonstrated: Critical comments on Gogel's "The sensing of retinal motion." Perception \& Psychophysics, 1981, 29, 170-172.

voN KrIEs, J. Notes on chapter 29. In J. P. C. Southall (Ed.), Helmholtz's treatise on physiological optics (Vol. 3). New York: Dover, 1962.

(Manuscript received August 1, 1983; accepted for publication August 5, 1983.) 